\title{
Leitbild und Handlungsfelder zur Öffnung des öffentlichen Einkaufs in Deutschland
}

Die ausführliche Bestandsaufnahme, die Ergebnisse der Online-Befragung und der Blick auf die Best Practices bilden die Grundlage, um in diesem Kapitel ein Leitbild für die Öffnung der öffentlichen Einkaufsdaten zu entwerfen. Bei Leitbildern

\begin{abstract}
„... handelt es sich um Umsetzungen von Visionen, mit denen ein in unbestimmter Zukunft vorstellbarer oder wünschbarer Zustand beschrieben werden soll. Visionen sollen als Leitideen Zuversicht und Aufbruchstimmung ausstrahlen, aber auch eine Herausforderung bedeuten und Änderungsbereitschaft signalisieren" (von Lucke, 2008, S. 21).
\end{abstract}

Anhand der Formulierung von Maßnahmen kann ein Leitbild weiter präzisiert und idealerweise auch in der Praxis wirksam werden. In Anlehnung an die vorhergehenden Kapitel erfolgt eine allgemeine Beschreibung des Leitbilds für die Öffnung der öffentlichen Einkaufsdaten, bevor die jeweiligen Handlungsfelder entlang der Wirkungsdimensionen Strategie und Organisation, Recht, Technologie, Transparenz, Partizipation und Kollaboration vertieft werden (siehe Abbildung 10.1). 


\section{Vision:}

Öffnung der öffentlichen Einkaufsdaten

als Teil der digitalen Transformation des öffentlichen Einkaufs

Mission: Öffnung der öffentlichen Einkaufsdaten des Unterschwellenbereichs

zur optimierten Steuerverwendung über das GovData-Portal für Deutschland

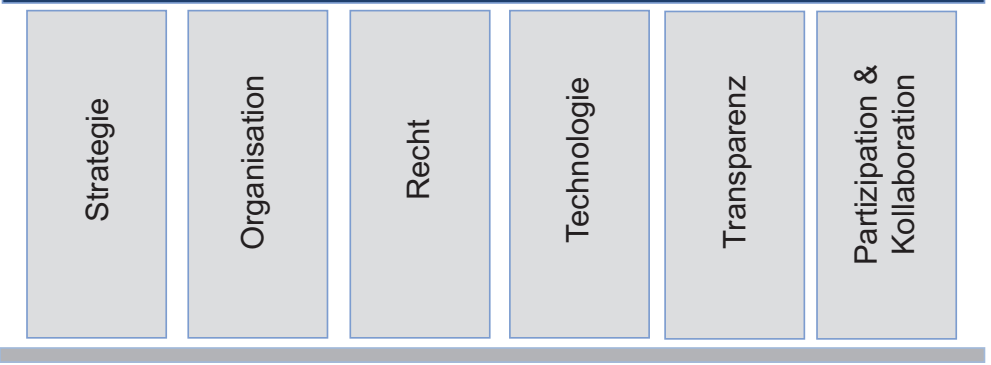

Abbildung 10.1 Leitbild der Öffnung öffentlicher Einkaufsdaten

\subsection{Vision}

Die oben angestellten Überlegungen konnten die Notwendigkeit der Transparenz im Unterschwellenbereich aufzeigen und die technologischen Möglichkeiten zur Öffnung des öffentlichen Einkaufs nachweisen. Die Literaturauswertung und die Online-Erhebung zeigen eine deutliche Befürwortung dieser Öffnung entlang des Gesamtprozesses des öffentlichen Einkaufs. Wenngleich das sinnvolle Ausmaß diskutiert und differenziert werden muss, wird insgesamt eine stärkere Öffnung gewünscht (siehe Abschnitt 8.3). Mit den Wünschen und Möglichkeiten hält die Entwicklung in diesem Bereich bisher nicht Schritt. Um dies zu ändern, kann zunächst die Beschreibung einer Vision hilfreich sein. Eine Vision stellt einen wünschenswerten Zustand mit einem Zeithorizont von etwa fünf bis zehn Jahren dar. Visionen sollten verständlich formuliert sein, holistisch ansetzen, messbare Ziele beinhalten und unterschiedliche Erwartungshaltungen vereinen (Kaczorowski, 2014, S. 186).

Als Teil einer übergeordneten Vision erscheint es sinnvoll, die Öffnung der öffentlichen Einkaufsdaten in das Konzept des urbanen Datenraums einzuordnen. Dieser Ansatz wird vielfach diskutiert, meist im Zusammenhang mit Datenwirtschaft, aber auch Smart Government und Smart-City-Ansätzen. Eine Datenwirtschaft umfasst die Gesamtheit personenbezogener und nicht-personenbezogener Daten des öffentlichen und privaten Sektors sowie den vollständigen Kreislauf von der Erhebung bis zur Löschung von Daten, welche verschiedene Akteure bedient (beispielsweise Unternehmen, Bürger, Wissenschaft) (Schieferdecker et al., 2018, 
S. 22). Datenräume beinhalten digitale Daten (zum Beispiel Rohdaten, Metadaten, Informationen) und ermöglichen digitale Dienste. Sie beinhalten eine Dateninfrastruktur, auf deren Basis dezentral Daten zwischen den Akteuren ausgetauscht und verknüpft werden können, es wird durch ein Netzwerk an Akteuren gestaltet und kann auch einen räumlichen Geltungsbereich haben. Es gibt Datenräume somit dezentral auf europäischer, nationaler, regionaler und lokaler Ebene, in Unterscheidung nach Industrie und Verwaltung sowie nach Domänen (zum Beispiel Mobilität, Energie oder Finanzen). Der urbane Datenraum enthält alle Daten, die für eine Kommune relevant sind und stellt somit die Datenbasis einer Smart City dar. Auf dieser Basis können weitere Anwendungen erstellt werden. Die Öffnung der Datenbestände ist dabei ein Ziel, jedoch geht es insbesondere um den Aufbau von domänen- und bereichsübergreifenden sowie horizontalen Diensten. Sie sollen dazu beitragen, dass kommunale Prozesse von Anfang bis Ende besser analysiert und organisiert werden können. Somit stellen Open Data-Portale und auch das GovData-Portal gemäß Tabelle 10.1 im Sinne von „Government as a Platform“ unter Open Government den Beginn dar, finden aber ihre Fortsetzung im Smart Government-Konzept, welches cyberphysische Systeme berücksichtigt und die Abwicklung von Prozessen mit intelligent vernetzten Informations- und Kommunikationstechniken unterstützt. Schließlich wird dies auf der Stufe des taktilen Internets um Echtzeitberechnungen im Rahmen des Real-Time Governments ergänzt (Barns, 2018, S. 13; Schieferdecker et al., 2018, S. 29 ff.; von Lucke, 2016, S. 8).

Tabelle 10.1 Häfler Stufenmodell für die weitere Entwicklung des World Wide Webs (in Anlehnung an von Lucke, 2016, S. 8)

\begin{tabular}{|l|l|l|l|}
\hline Web & Beschreibung & $\begin{array}{l}\text { Art der } \\
\text { Kommunikation }\end{array}$ & Stufe \\
\hline Web 5.0 & Taktiles Internet & $\begin{array}{l}\text { Netzwerkkommunika- } \\
\text { tion nahezu in Echtzeit }\end{array}$ & Real-Time Government \\
\hline Web 4.0 & $\begin{array}{l}\text { Internet der Dinge und } \\
\text { Internet der Dienste }\end{array}$ & $\begin{array}{l}\text { Smarte Objekte, cyber- } \\
\text { physische Systeme }\end{array}$ & Smart Government \\
\hline Web 3.0 & $\begin{array}{l}\text { Internet der Daten, } \\
\text { Semantisches Web }\end{array}$ & $\begin{array}{l}\text { Linked Data, Open } \\
\text { Data, Big Data, Big } \\
\text { Data Analytics }\end{array}$ & Open Government Data \\
\hline Web 2.0 & $\begin{array}{l}\text { Internet der Menschen, } \\
\text { Internet zum } \\
\text { Mitmachen }\end{array}$ & $\begin{array}{l}\text { Netzwerkkommunika- } \\
\text { tion über Social Media }\end{array}$ & Open Government \\
\hline Web 1.0 & $\begin{array}{l}\text { Internet der Systeme, } \\
\text { World Wide Web }\end{array}$ & $\begin{array}{l}\text { Netzwerkkommunika- } \\
\text { tion über das World } \\
\text { Wide Web }\end{array}$ & Electronic Government \\
\hline & & & \\
\hline
\end{tabular}


Somit würde die Öffnung öffentlicher Einkaufsdaten als Teil des GovData-Portals einen dezentralen urbanen Datenraum darstellen. Dieser Datenraum kann durch Erweiterung - zum Beispiel mittels funktionsübergreifender Verknüpfung mit anderen Datenbeständen, die wiederum cyberphysisch verbunden sein können - zusätzliches Potential entfalten. Dies können zum Beispiel die Objekte einer Smart City-Infrastruktur wie Straßenlaternen, Kameras oder Einrichtungen des Gesundheitswesens sein (Tenderlake Ltd., 2019b). Schließlich werden Echtzeitkalkulationen weiteren Mehrwert generieren (von Lucke, 2019, S. 50 ff.).

Angewendet auf den öffentlichen Einkauf könnte ein „digitaler Vertrag“ unmittelbar Informationen zur Leistungsfähigkeit beschaffter und eingebundener smarter Objekte erhalten - bei einem Ausfall oder bereits proaktiv mithilfe künstlicher Intelligenz durch Mustererkennung könnte automatisiert und rechtssicher für definierte Bereiche eine Neubeschaffung angestoßen werden (unter Nutzung sogenannter ,Smart Contracts ${ }^{1 “}$ ). In anderen Bereichen könnten Angebote automatisiert auf unterschiedliche Kriterien (Preis, Korruptionsrisiken etc.) hin ausgewertet und eine Vorauswahl passender Bieter vorgenommen werden, wie auch einige Best Practices bereits aufzeigen konnten. So konstatiert Hill:

„Bei Auftragsvergabe-, Verteilungs- oder Koordinierungsaufgaben der Verwaltung, etwa im Bereich der Wirtschaftsverwaltung oder der Daseinsvorsorge, kann KI regel- und gleichheitsorientierte Lösungen bieten und Optimierungsvorschläge (...) entwickeln“ (Hill, 2018b, S. 289).

Diese neuen technischen Möglichkeiten erfordern allerdings auch eine bewusste Auseinandersetzung damit, welche Prozesse und Entscheidungen automatisiert und durch KI getrieben werden sollten und wo der menschliche Ermessensspielraum wichtig ist (Hill, 2018b, S. 290). Es gilt also, KI im Kontext des öffentlichen Einkaufs zu hinterfragen und an geeigneter Stelle einzubinden. So könnte sich der öffentliche Einkauf zum intelligenten Steuerer entwickeln.

Eine weitere Dimension betrifft unterschiedliche Bereitstellungsformen wie in Abbildung 10.2 dargestellt. So werden öffentliche Einkaufsdaten aktuell nur im begrenzten Rahmen offener Verwaltungsdatenportale als Data Repositories bereitgestellt, obwohl sie gleichermaßen im Rahmen von sogenannten Data

\footnotetext{
${ }^{1}$ „Smart Contracts“ sind digitale Verträge, die auf Computerprotokollen und der Blockchain-Technologie aufbauen. Sie bieten eine effizientere Abwicklung gegenüber herkömmlichen Verträgen. Eine bekannte Plattform, die smarte Verträge anbietet, ist Ethereum (Schiller, 2019).
} 


\begin{tabular}{|c|c|c|c|}
\hline Data Repositories & Data Showcases & City Scores & Data Marketplaces \\
\hline $\begin{array}{l}\text { Offene Datenportale } \\
\text { - Bieten Zugang zu Regie- } \\
\text { rungsdaten in zumeist } \\
\text { maschinenlesbaren } \\
\text { Formaten } \\
\text { - Die Daten sind normaler- } \\
\text { weise nicht ausgerichtet an } \\
\text { Vorgaben oder Leistungs- } \\
\text { zielen } \\
\text { - Erstellt durch Verwaltungen }\end{array}$ & $\begin{array}{l}\text { City Dashboards } \\
\text { - Bieten Zugang zu Daten- } \\
\text { visualisierungen in Abstim- } \\
\text { mung mit städtischen } \\
\text { Prioritäten } \\
\text { - Die hierfür genutzten Daten } \\
\text { sind nicht immer erhältlich } \\
\text { oder maschinenlesbar } \\
\text { - Erstellt durch Verwaltungen } \\
\text { oder in Zusammenarbeit } \\
\text { mit Forschungsinstitutionen }\end{array}$ & $\begin{array}{l}\text { Score Cards } \\
\text { - Integrieren mehrere Daten- } \\
\text { sätze, um so Leistungs- } \\
\text { messungen gegen konkrete } \\
\text { Ziele zu ermöglichen } \\
\text { - Die genutzten Daten sind } \\
\text { nicht immer erhältlich } \\
\text { - Erstellt durch Verwaltungen }\end{array}$ & $\begin{array}{l}\text { Data Stores } \\
\text { - Bieten Zugang zu } \\
\text { maschinenlesbaren Daten } \\
\text { - Datenzugang und -nutzung } \\
\text { durch externe Parteien wird } \\
\text { unterstützt (inklusive der } \\
\text { Weitervermarktung) } \\
\text { - Leistungsmessung ist eine } \\
\text { von vielen möglichen } \\
\text { Anwendungen } \\
\text { - Erstellt durch Verwaltungen } \\
\text { oder den privaten Sektor }\end{array}$ \\
\hline $\begin{array}{l}\text { Ziele } \\
\text { - Innovationen rund um } \\
\text { Datendienste } \\
\text { - Transparenz }\end{array}$ & $\begin{array}{l}\text { Ziele } \\
\text { - Datenvisualisierungen } \\
\text { - Transparenz }\end{array}$ & $\begin{array}{l}\text { Ziele } \\
\text { - Leistungsmessung }\end{array}$ & $\begin{array}{l}\text { Ziele } \\
\text { - Innovationen rund um } \\
\text { Datendienste }\end{array}$ \\
\hline
\end{tabular}

Abbildung 10.2 Urbane Datenplattformen (in Anlehnung an Barns, 2018, S. 7, übersetzt durch Verfasserin)

Showcases, City Scores und Market Places einsetzbar wären, wo weitere Anwendungsmöglichkeiten offenständen (Barns, 2018, S. 7).

Innerhalb der Dimensionen Bereitstellungsstufen und Bereitstellungsformen (siehe Abbildung 10.3) können dann die Geschäftsideen aus der durchgeführten Studie Berücksichtigung finden (siehe Abschnitt 8.2.2; Fragen 19 und 20).

Die Vision könnte somit abstrakt formuliert wie folgt lauten:

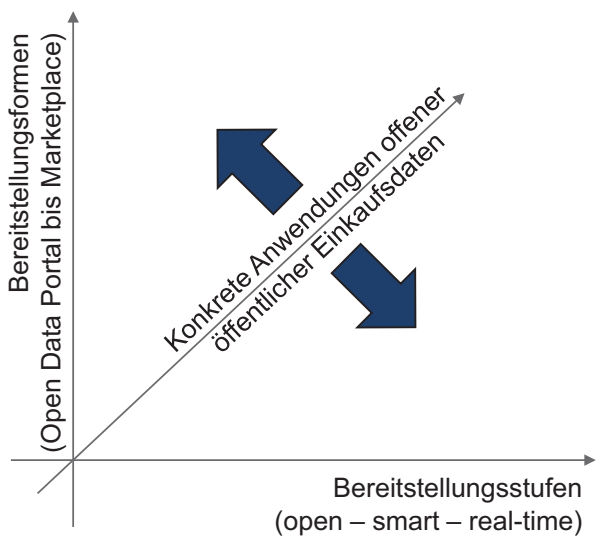

Abbildung 10.3 Einordnung der Vision öffentlicher Einkaufsdaten zwischen Bereitstellungsstufen und -formen 
Spätestens im Jahr 2030 werden öffentliche Einkaufsdaten - also die für den Oberschwellenbereich und insbesondere jene für den Unterschwellenbereich zentral über das GovData-Portal als eigener urbaner Datenraum („Public Procurement Data Space") als Dokument und maschinenlesbar angeboten. Gleichzeitig stehen Auswertungen und Visualisierungsoptionen bereit, die einer breiten Öffentlichkeit die eigenständige und benutzerfreundliche Analyse der Daten ermöglichen. Zudem ist die Kombination öffentlicher Einkaufsdaten mit anderen Daten als LOD sowie Echtzeitauswertungen über fehlerhafte Verfahren oder Preise im Verhältnis zur vergangenen und aktuellen Lieferantenperformance möglich. Die offenen öffentlichen Einkaufsdaten stehen zusätzlich in digitalen Marktplätzen oder als Bestandteil von City Scores zur Verfügung. Ergänzt wird das Angebot öffentlicher Einkaufsdaten in festgelegten Bereichen durch smarte Verträge. Insgesamt wird so eine Kontrolle staatlicher Ausgaben durch Dritte möglich. Es entstehen neue Geschäftsmodelle und Unternehmen können einfacher an Ausschreibungen teilnehmen. Die erzielten Einsparungen erleichtern nachweislich die Investition von Steuermitteln in anderen Bereichen, was insgesamt zu einer höheren Glaubwürdigkeit der Politik und Zufriedenheit der Bürger führt.

Um diese Vision verwirklichen zu können, ist die Erarbeitung verschiedener Handlungsfelder nötig, auf die nun eingegangen wird.

\subsection{Handlungsfelder}

\subsubsection{Strategie}

Um einen sichtbaren Fortschritt zur Öffnung des öffentlichen Einkaufs im Unterschwellenbereich zu erzielen und den verschiedenen Anforderungen unterschiedlicher Zielgruppen gerecht zu werden, erscheint es sinnvoll, in zwei Richtungen $\mathrm{zu}$ arbeiten.

1. Verankerung der Öffnung öffentlicher Einkaufsdaten in den politischen Programmen, Aufnahme in den dritten NAP der OGP sowie Teilnahme Deutschlands in der Initiative „Open Contracting Partnership“

Zunächst sollte die Öffnung öffentlicher Einkaufsdaten in den politischen Programmen Deutschlands fest verankert werden, zum Beispiel in der Fortführung der „Digitalen Verwaltung“ oder in der „NEGS“. Die aktuellen Programme sind bis circa 2020 ausgelegt (siehe Abschnitt 5.2.3). Sobald es an die Definition des nächsten Fünfjahreszeitraums geht, sollte die Öffnung 
des öffentlichen Einkaufs dort aufgenommen werden. Gleichzeitig sollte sich Deutschland in den relevanten NGOs stärker positionieren. Der Forderung nach Öffnung des öffentlichen Einkaufs im NAP (Open Government Deutschland e. V., 2019; Open Government Partnership Deutschland e. V., 2019, S. 16 f.) sollte spätestens mit dem dritten NAP im Jahr 2021 nachgekommen werden - umso mehr, da Deutschland nun auch Teil des Lenkungsausschusses ist und damit eine Vorbildfunktion einnehmen sollte. Der Konsultationsprozess sollte breiter angelegt und auf Teilnehmer der Wirtschaft, Wissenschaft und Medien ausgeweitet werden. Damit einhergehend sollte Deutschland ebenfalls Mitglied in der OCP werden (Open Government Partnership Deutschland e. V., 2019, S. 16 f.). Um allein diesen Schritt zu erreichen, kann es im Vorfeld erforderlich sein, das Bewusstsein der politischen Vertreter für dieses Thema mithilfe verschiedener Gremien zu schärfen, damit ein offener öffentlicher Einkauf nicht nur ein Schlagwort bleibt (Granickas, 2016, S. 10).

\section{Aufsetzen eines interdisziplinären und ebenenübergreifenden Projekts zur Öffnung öffentlicher Einkaufsdaten}

Darüber hinaus könnte ein interdisziplinär besetztes Projekt zur Öffnung des öffentlichen Einkaufs sinnvoll sein, welches die strategischen und operativen Fragestellungen vorbereitet, zur Entscheidung und in die Umsetzung bringt. Dieses Projekt sollte Teilnehmer aus verschiedenen Zielgruppen (Wirtschaft, Verwaltung, Wissenschaft, NGOs, Politik, Medien, Bürger - siehe Abschnitt 5.4) und föderalen Ebenen (Bund, Länder, Kommunen) integrieren, um möglichst frühzeitig eine umfassende Perspektive auf das Thema zu gewinnen. Dies wird auch in Erfahrungsberichten als Vorteil eingestuft: „In open contracting and public spending, a collaborative partnership between government, civil society and the private sector is often referred to as the "golden triangle" (Granickas, 2016, S. 13). Da die stark fragmentierte kommunale Ebene einen Anteil von bis zu 40-60 \% am gesamten Beschaffungsvolumen trägt (Beck \& Schuster, 2013, S. 8; Bundesministerium des Innern \& Bundesministerium für Wirtschaft und Arbeit, 2004, S. 3), sollte diese besonders berücksichtigt werden. Dieses Projekt sollte von einer gemeinsamen Stelle koordiniert werden. Diese könnte auf Bundesebene, aber auch bei einem Land angesiedelt sein, welches dann die übergreifende Projektsteuerungsaufgabe übernimmt.

Die Teilnahme und das Projektvorgehen müssen abgestimmt und zugesichert sein. Der zeitliche Umfang der Teilnahme sollte klar benannt werden, um Teilnehmer nicht zu frustrieren und im Verlauf des Projekts zu verlieren. Als Projektvorgehensweise bieten sich neben traditionellen Vorgehensweisen auch agile Methoden an. Da es sich beim öffentlichen Einkauf um ein komplexes Thema 
handelt, kann mit Agilität der Vielfalt und den stetigen Veränderungen möglicherweise besser begegnet werden (Hill, 2015, S. 416). Denkbar ist ein stetiger Fortschritt, der sich an kürzeren Teilprojektphasen orientiert. Weiters muss festgelegt werden, in welcher Reihenfolge der Anbindung welcher Datenquellen welche Daten über den Projektverlauf veröffentlicht werden. Ebenfalls wichtig erscheint ein Kommunikationskonzept, welches einerseits regelmäßig über den Projektfortschritt informiert, andererseits kontinuierliche Teilhabe im Sinne von Partizipation und Kollaboration mit Multiplikatoren des Projektes sowie der breiten Öffentlichkeit zulässt.

\subsubsection{Organisation}

\section{Besetzung des Projektes mit verschiedenen Akteuren}

Es erscheint sinnvoll, dass unterschiedliche Organisationen des öffentlichen Einkaufs in dem unter Handlungsfeld 2 skizzierten gemeinsamen Projekt vertreten sind. Hier sollten sowohl Teilnehmer mit ersten Erfahrungen in der Öffnung öffentlicher Verwaltungsdaten, idealerweise Einkaufsdaten, als auch Teilnehmer, für die dies noch Neuland ist, aufgenommen werden. Mit Blick auf die föderale Verankerung (Bund, Land, Kommune) könnten dies zum Beispiel jene Bundesund Landesbehörden sein, die bereits als Einkaufskooperationen organisiert sind (siehe Abschnitt 6.3.1), Vergabestellen unterschiedlicher Größen, Vertreter von GovData als zentralem Datenportal, der bestehenden Transparenzportale (zum Beispiel Hamburg, Bremen) oder der Vorreiter-Kommunen (zum Beispiel Köln, Bonn). Weiterhin denkbar sind Teilnehmer statistischer Ämter oder der Rechnungshöfe.

Aus der Wirtschaft sollten sowohl Anbieter und Betreiber von Vergabe- und Bekanntmachungsportalen als auch Vertreter von Organisationen, die regelmäßig an Ausschreibungen der öffentlichen Hand teilnehmen (zum Beispiel Verbände, aber auch Unternehmen selbst), in Betracht kommen. Ebenso relevant erscheinen Vertreter querschnittlicher (Nichtregierungs-)Organisationen oder Projekte, die sich bereits konkret mit öffentlichen Einkaufsdaten auseinandersetzen oder auseinandergesetzt haben (zum Beispiel „Code for Germany“, „Digiwhist“).

Die Auswahl der richtigen Vertreter im Sinne einer repräsentativen Mischung und einer offenen, konstruktiven Einstellung und ihre möglichst frühe Beteiligung scheint eine wichtige Voraussetzung für den Projekterfolg zu sein. 


\section{Definition und Nutzung von Standards}

Ein weiterer Aspekt der Organisation ist die Entscheidung gegen oder für die Nutzung von Standards als Grundlage für das Projekt. Standards bieten den Vorteil, dass sie die Sammlung und Strukturierung öffentlicher Einkaufsdaten, ihre Weiterverwendung sowie das Monitoring vereinfachen (siehe Abschnitt 7.2.1). Der OCDS als aktuell einziger internationaler Standard sollte mit Blick auf ein Musterdatenschema sowie die Anwendbarkeit des Vorgehens für Deutschland überprüft und - falls erforderlich - angepasst werden (Arbeitskreis Open Government Partnership Deutschland, 2017, S. 42; Granickas, 2016, S. 8). Dies sollte als Teil der Bestandsanalyse erfolgen (siehe Handlungsfeld 10). Die Nutzung von Standards wird als Best Practice auch in der Online-Befragung genannt (siehe Abschnitt 8.3.3, Frage 36).

\section{Erarbeitung von Geschäftsmodellen und User Stories pro Anwender- gruppe}

Als Teil der gemeinsamen Arbeit sollten Geschäftsmodelle, praktische Beispiele und Anwendererzählungen (sogenannte „User Stories“) entwickelt werden, die die quantitativen und qualitativen Mehrwerte benennen. So können die wichtigsten Themen identifiziert und umgesetzt werden, was die Gefahr reduziert, sich in der Vielfalt der möglichen Aspekte zu verlieren. Gleichzeitig werden auf diese Weise die Bedarfe der einzelnen Akteure noch einmal konkret adressiert. Dies unterstützt eine der zentralen Anforderungen aus dem finalen Workshop (siehe Abschnitt 8.1.6). Die User Stories sollten von einer Kosten-/ Nutzenschätzung begleitet werden, die im Projektverlauf an Genauigkeit gewinnt. Gleichzeitig können Verwaltungen so frühzeitig Vorschläge zur Deckung der Kosten in Form von Geldleistungsmodellen erarbeiten.

\subsubsection{Recht}

\section{Ausweitung der Berichtspflichten auf den Unterschwellenbereich und den gesamten öffentlichen Einkaufsprozess im Recht sowie in der Statistik}

Das Vergaberecht wird über Bund, Länder und Kommunen in unterschiedlichen Regelungen umgesetzt. Dies ist der föderalen Struktur geschuldet, weshalb es eine besondere Herausforderung ist, auf Vereinheitlichungen „,von oben“ hinzuwirken. Dies macht nochmals den Vorteil einer gemischten Projektbesetzung möglichst aller föderalen Ebenen deutlich, da so die Themen adressatengerecht in unterschiedlichen Gremien eingesteuert werden können. 
Aktuell gibt es nur wenige Veröffentlichungspflichten für den Unterschwellenbereich (siehe Abschnitt 6.1). Die Einschränkungen orientieren sich im Wesentlichen an anderen Rechtsgebieten wie dem Datenschutz oder dem Informationsfreiheitsgesetz. Eßig ergänzt:

„So lange die oben skizzierten Prinzipien der ordnungsgemäßen Vergabe eingehalten und das vorgeschriebene Vergabeverfahren [die Phasen Ausschreibung und Vergabe betreffend, Anm. d. Verf.] fehlerfrei durchgeführt wird, steht es der öffentlichen Beschaffung frei, alle skizzierten Elemente des strategischen Beschaffungsprozesses zu bearbeiten“"(Eßig, 2008, S. 301).

Dies lässt also einen Handlungsspielraum zu, den man für die Öffnung des öffentlichen Einkaufs nutzen sollte, wie die Städte Hamburg und Bremen bereits zeigen. Für das IFG-Bund ist es so, dass öffentliche Einkaufsdaten bereits durch den Begriff der ,amtlichen Informationen“ abgedeckt sind. Demnach müssen öffentliche Einkaufsdaten gar nicht immer explizit erwähnt sein, um sie anfordern zu können (Open Contracting Partnership, 2019e, S. 5 f.). Gleichzeitig sollten die Definitionen rund um Betriebs- und Geschäftsgeheimnisse und personenbezogene Daten geschärft werden (siehe hierzu auch Abschnitt 10.2.5, Handlungsfeld 13).

Unstrittig erscheint, dass eine stärkere Verpflichtung zur Veröffentlichung im Unterschwellenbereich über die Verankerung im Recht eine größere Sogwirkung für die Veröffentlichungen hätte. Die eVergabe hat eindrücklich gezeigt, dass das Setzen auf Freiwilligkeit nicht zu maßgeblichen Fortschritten geführt hat (siehe Abschnitt 6.2.1.10). Die Ausweitung der verpflichtenden eVergabe auf den Baubereich, eine insgesamt breitere Veröffentlichungspflicht für den Unterschwellenbereich mit einer festzulegenden Mindestschwelle in Euro sollten im Vergaberecht, aber auch in der VergStatVO verankert werden. Dies muss einerseits alle Phasen des Einkaufs (von der Planung bis zur Ausführung) (siehe Abschnitt 6.3.2) und andererseits ein umfangreicheres Datenspektrum (siehe Abschnitt 6.3.2 und Abschnitt 7.3.9) betreffen. Die Anregungen im finalen Workshop (siehe Abschnitt 8.1.6) und die Ergebnisse der Online-Befragung (siehe Abschnitt 8.2.3, Fragen 33 und 34) untermauern dies ebenfalls. Weiters könnte diskutiert werden, einen weiteren Bekanntmachungsstatus einzuführen: Neben der Ausschreibungs- und Vergabebekanntmachung könnte dies eine Projektabschlussinformation sein, sodass ad hoc auswertbar wäre, welche Projekte in welchem Zeitraum bereits umgesetzt wurden.

Parallel sollte man nicht nur auf eine konsolidierte, veränderte Rechtsprechung warten, sondern auf der Basis der bestehenden Rechtsvorschriften einen Lösungsraum erarbeiten. Das Warten kann andernfalls zulasten konkreter 
Ergebnisse in einer überschaubaren Zeit gehen. In diesem Fall erscheint die ausgearbeitete Argumentation über Geschäftsmodelle sowie mehrwertige Anwendungsbeispiele (siehe Abschnitt 10.2.2) hilfreich.

Es sollte auch darauf hingewirkt werden, die statistischen Ämter in der Rolle der Datenbereitstellung zu unterstützen, da die Aufbereitung und Plausibilisierung von Daten bereits ihr tägliches Kerngeschäft sind. Wie im Beispiel gezeigt: Das Statistikamt in der Slowakei ist der Hauptlieferant von Daten für das slowakische Open Data-Portal (Office of the Plenipotentiary of the Government of the Slovak Republic for the Development of the Civil Society, 2017, S. 5).

\section{Definition öffentlicher Einkaufsdaten als „,High Value Data Set“}

Es gilt vor dem Hintergrund der neuen PSI-Richtlinie (EU 2019/1024) die Definition öffentlicher Einkaufsdaten als „High Value Data Set“ unter „Statistik“ in der nationalen Umsetzung der neuen Richtlinie zu berücksichtigen (siehe Abschnitt 7.1.1). Um den Interpretationsspielraum einzugrenzen, ist es sinnvoll, Begriffe wie „Betriebs- und Geschäftsgeheimnisse“ zu präzisieren, so wie dies bereits partiell (zum Beispiel vom InGFA) eingefordert wurde (siehe Abschnitt 7.1.3.1). Die erarbeiteten Ergebnisse sind in die jeweiligen Gremien der unterschiedlichen Ebenen einzubringen.

\subsubsection{Technologie}

\section{Zentrale Veröffentlichung öffentlicher Einkaufsdaten auf dem GovData- Portal}

Technologisch gibt es mit dem GovData-Portal bereits einen soliden Ansatzpunkt. Dort können auch öffentliche Einkaufsdaten zentral und im Sinne der Definition von „open“ eingestellt werden. Die Umfrage förderte den klaren Wunsch der Befragten nach zentraler Bereitstellung zutage (siehe Abschnitt 8.2.2, Frage 17). Der Prozess der Bereitstellung müsste im Rahmen des Projektes erarbeitet werden. Die Angebote zur Interaktion, also zur Partizipation und Kollaboration, sind bei dem GovData-Portal insgesamt noch ausbaufähig. Es sollten nicht nur verschiedene Formate (Online-Lab, Forum, Feedbackformen etc.), sondern auch Werbung in der Öffentlichkeit zur Nutzung des Portals in Betracht gezogen werden, um es von einem datenzentrierten zu einem nutzerzentrierten Portal weiterzuentwickeln (OECD, 2018b, S. 170 f.). Interessant für den öffentlichen Einkauf sind die geplanten Erweiterungen der Plattform in Richtung LOGD sowie Auswertungs- und Visualisierungsmöglichkeiten (siehe Abschnitt 7.3.9.2). 
Darüber hinaus sollten perspektivisch Technologien wie Blockchain und KI berücksichtigt werden. Ein Beispiel hierfür ist Kanada, das mithilfe von Blockchain-Technologie die Registrierung der Lieferanten im öffentlichen Einkauf vorantreiben möchte. Die Ausgangssituation ist, dass Regierungen, Bürger und Unternehmen häufig die gleichen Informationen anfragen (zum Beispiel Name, Adresse). Die Überprüfungen finden mehrfach und wiederholt über unterschiedliche Kanäle (online, offline, Papier) statt, obwohl die Informationen bereits bei anderen Behörden vorliegen. Bedingt durch technische und rechtliche Vorgaben gibt es somit eine Vielzahl einzelner und verteilter Datenhaltungen. Mithilfe von Blockchain soll es möglich werden, dass die Daten nur einmalig erhoben (,tell us once") und dann automatisch bei zusätzlichen Anfragen behördenübergreifend weiterverwendet werden können (,,validate once“) (Serghi, 2018).

\section{Verschränkung von technologie-/entwicklungsaffinen sowie anwender- orientierten Rollen in der Ausarbeitung der Konzepte}

Als Mitwirkende sind einerseits die technischen Rollen interessant wie zum Beispiel Architekten, Solution Consultants, Datenanalysten und Entwickler. Andererseits ist aber auch die Integration der Nutzerperspektive durch die Anwender der Daten essentiell, da eine Technologie nur dann ihre Stärke entfaltet, wenn sie tatsächlich genutzt wird. Die Nutzer sollten unterschiedlichen Gruppen entstammen, damit ein möglichst breites Feedback berücksichtigt werden kann (siehe Abschnitt 10.2.6). Wie der Prozess des Feedbacks organisiert sein könnte, ist unter Handlungsfeld 14 in Abschnitt 10.2.6 spezifiziert.

\section{Bestandsaufnahme und Entwicklung eines Musterdatenschemas}

In einer Bestandsaufnahme sollten bestehende Systeme, verfügbare Dokumente und Daten, ihre Unterschiede und die aktuelle Datenqualität im Vordergrund stehen. Die realen Daten müssen mit dem in Abschnitt 10.2.2 festgelegten Standard abgeglichen werden (Arbeitskreis Open Government Partnership Deutschland, 2017, S. 42). Gegebenenfalls machen sie Anpassungen des Standards für Deutschland erforderlich. Der Abgleich zwischen dem Standard und der Realität hilft, nach und nach das für Deutschland passende Musterdatenschema als Teil eines Sollkonzepts zu entwickeln und anzuwenden. Die Ergebnisse könnten dann in das Projekt zur Vergabestatistik genauso einfließen wie in die Öffnung der öffentlichen Einkaufsdaten über GovData.

\section{Erarbeitung des Sollkonzepts inklusive der Verankerung von Datenqualität}

Das Sollkonzept beinhaltet neben dem Musterdatenschema künftige Prozesse der Datenbereitstellung, der -plausibilisierung und -bereinigung sowie Auswertungs- und 
Visualisierungsmöglichkeiten. Datenqualität herzustellen und aufrechtzuerhalten ist essentiell für eine belastbare Transparenz (Mendes \& Fazekas, 2018, S. 21 f.). Ein klarer Prozess mit Verantwortlichen (und gegebenenfalls die Einführung der Rolle eines „General Data Administrators“ wie in Frankreich, siehe Abschnitt 9.2.2.4) sowie eine regelmäßige Kontrolle können hier unterstützen. Darüber hinaus sind Innovationsthemen wie LOD und KI für den öffentlichen Einkauf zu bewerten (Arbeitskreis Open Government Partnership Deutschland, 2017, S. 42; Mendes \& Fazekas, 2018, S. 19). Gegebenenfalls kann ein Pilot entwickelt werden, der erste Anwendungsmöglichkeiten aufzeigt und den Nutzern hilft, konstruktives Feedback zu geben.

\subsubsection{Transparenz}

\section{Transparenz im Spannungsfeld einer veränderten Fehlerkultur und nachweislich zu lösenden Problemen}

Die Befragungsergebnisse zeigen in Bestätigung der theoretischen Erkenntnisse aus der Literatur, dass eine der größten Stärken der Öffnung öffentlicher Einkaufsdaten die Erhöhung der Transparenz ist (92\% Zustimmung), insbesondere im Umfeld der Verfahrens-, Anbieter- und Lieferantentransparenz (78\% Zustimmung), unmittelbar gefolgt von einer Verminderung der Korruption (86\% Zustimmung), primär in den Teilprozessen der Ausschreibung/Bekanntmachung, Vergabe und Kontrolle. Als größte Chance wird die Korruptionsprävention mit $83 \%$ Zustimmung angesehen (siehe Abschnitt 8.2.2, Fragen 23 und 25 und Abschnitt 8.2.3, Fragen 27 und 29). Für die Befragten ist der positive Zusammenhang zwischen Transparenz und Korruptionsreduktion somit eindeutig. Auch die erörterte Literatur gibt hierzu Belege (siehe Abschnitt 6.5.1).

Im finalen Workshop (siehe Abschnitt 8.1.6) wurde thematisiert, dass Transparenz oft angstbesetzt sei, da man hierunter zuvorderst „Anprangern“ und „Vorführen“ verstehe. Wichtig sei, anstelle von „bashing“ oder „naming und shaming“ in einen kritischen Diskurs zu treten. Es sollten viel stärker die Optimierungsmöglichkeiten und Chancen in den Vordergrund gerückt werden. Dies verdeutlicht, dass der Umgang mit Fehlern eine gezielte Auseinandersetzung benötigt, unter Umständen sogar die bestehende Kultur auf den Prüfstand stellt. Mögliche erste Fragen, die diesen Diskurs treiben, könnten sein: Wie ist die aktuelle Fehlerkultur? Was ist die gewünschte Fehlerkultur? Was wird akzeptiert und wo werden Grenzen gezogen? Wandelt sich dies mit dem zeitlichen Verlauf des Projekts und zunehmendem Wissensaufbau der Beteiligten? 
Eine interessante Anregung war, über das „Drehen der Spielregeln“ ein Umdenken in Gang zu setzen, welches Transparenz sukzessive zu einer Selbstverständlichkeit werden lässt: Nicht die Transparenz der Daten benötigt eine Berechtigung, sondern ihre Geheimhaltung. Dies wurde auch in der Online-Befragung durch die Forderung unterstützt, dass das Gemeinwohl Vorrang vor unbegründeten Geheimhaltungsinteressen haben sollte (siehe Abschnitt 8.3.3, Frage 34). Zugleich wurde nochmals betont, dass Transparenz an sich nicht das Ziel sei, sondern Mittel zum Zweck. Mit der gewonnenen Transparenz müssten konkrete Probleme gelöst oder Optimierungen erreicht werden. Dies könnte Bestandteil der bereits erwähnten Anwendererzählungen („User Stories“) werden (siehe Abschnitt 10.2.2, Handlungsfeld 5).

\section{Definition eines stufenweisen Vorgehens zur Öffnung der Daten sowie Festlegung der zu öffnenden Datentypen, Warengruppen und Projekte}

Das Handlungsfeld adressiert, welche Daten wann und wie und für wen bereitgestellt werden. Dies kann sehr unterschiedlich sein, je nach Art der Daten und Geheimhaltungspflichten (zum Beispiel nationale Sicherheit) und je nachdem, zu welchem Zeitpunkt die Daten vorliegen und wann sie in welchem Rhythmus der Aktualisierung bereitgestellt werden und für welche Interessensgruppen (nicht alles ist für alle wichtig/richtig).

Im Vordergrund sollte die Ausgewogenheit zwischen Umfang der Daten und Umsetzbarkeit des Vorgehens stehen, da zu viele Daten möglicherweise erste Umsetzungsbeispiele verkomplizieren und verlangsamen. Es bietet sich an, zunächst mit Basisdaten zu beginnen und diese kontinuierlich auszubauen, dem Grundsatz „build a simple system and let it evolve“ (O'Reilly, 2011, S. 21) folgend und gemäß dem stufenweisen Vorgehen der OCP in Abbildung 10.4 (Open Contracting Partnership, 2019i). Ähnlich dem Fünf-Sterne-Schema

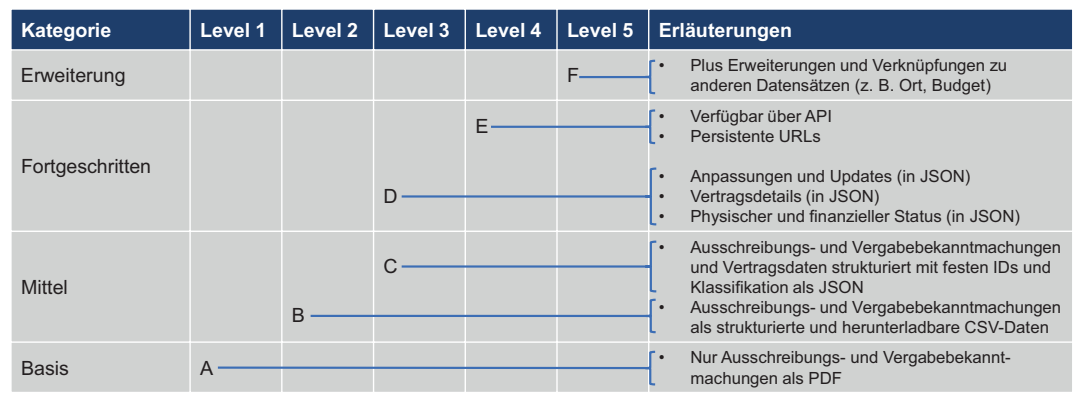

Abbildung 10.4 Grade der Veröffentlichung (in Anlehnung an Open Contracting Partnership, 2019i, übersetzt durch Verfasserin) 
von Berners-Lee (Berners-Lee, 2006, S. 6) sieht dies ebenfalls fünf Stufen der Öffnung für öffentliche Einkaufsdaten vor.

Weitere Möglichkeiten der Abschichtung könnten die schrittweise Öffnung der Daten nach Empfänger sein (zum Beispiel Öffnung gegenüber der Wissenschaft vor Öffnung gegenüber der breiten Öffentlichkeit) oder die Abschichtung nach Sektoren (zum Beispiel allgemeine öffentliche Einkaufsdaten vor Verteidigung und Sicherheit) oder Projekten (Projekte mit hoher Bedeutung für die Region vor Projekten unterhalb eines bestimmten Volumens). Das eröffnet die Chance, sich von kleineren zu größeren Empfängergruppen zu steigern oder sich mit zunehmendem Fach- und Projektverständnis von weniger komplexen bis hin zu komplexeren Datenbeständen zu entwickeln.

Die Studienergebnisse (siehe Abschnitt 8.2) geben hierzu ebenfalls Anregungen. Die Antworten auf Frage 14 legen nahe, dass nicht alle Akteure gleichermaßen Zugriff auf alle Daten haben sollten. Auf Basis dieser Angaben könnte der Zugang zu offenen öffentlichen Einkaufsdaten wie exemplarisch in Tabelle 10.2 skizziert geschaffen werden. In die Tabelle wurden die Studienergebnisse aus Frage 14 in die Kategorien „Zugang“, „kein Zugang“ und ,teils-teils“ überführt. Auch dies sollte Bestandteil einer vertiefenden Diskussion sein. Frage 15 konstatiert, dass Daten für Projekte mit einer hohen beziehungsweise negativen Außenwirkung mit $75 \%$ Zustimmung für eine Öffnung der Daten an erster Stelle stehen, und zwar noch vor „Projekten unterhalb eines bestimmten Volumens" (36\%) und ,allen Projekten“ (45\%). Hier ist eine Abwägung zwischen punktueller und struktureller Offenheit wichtig. Frage 16 zeigt bezüglich der Einschränkung von Daten bestimmter Sektoren oder Warengruppen eine etwa gleich hohe Anzahl von Ablehnungen und Befürwortungen, sodass dort (und vor allem mit Blick auf Verteidigung und Sicherheit) noch grundsätzlicher Abstimmungsbedarf besteht.

Es muss betont werden, dass die Antworten auf all diese Fragen kein ,entweder - oder" sein können, sondern eine Differenzierung erfordern: Ein Vertrag besteht nicht ausschließlich aus sensitiven Informationen und es bedarf einer genauen Prüfung und auch Definition, welche Informationen aufgrund Personenbezugs, Geschäfts- und Betriebsgeheimnissen ${ }^{2}$ oder nationaler Sicherheitsbedenken schützenswert sind. Diese Differenzierung sollte auch in die geltende Rechtsprechung einfließen.

${ }^{2}$ Die OCP gibt zur möglichen Differenzierung von Betriebs- und Geschäftsgeheimnissen eine gute Anregung, wie dies aussehen könnte (Open Contracting Partnership, 2019e, S. 5 f.). 
Tabelle 10.2 Zukünftiger Zugriff auf offene öffentliche Einkaufsdaten (basierend auf der Studie, Frage 14)

\begin{tabular}{|c|c|c|c|c|}
\hline \multirow[b]{2}{*}{ Planung } & \multicolumn{4}{|c|}{ Akteure } \\
\hline & $\begin{array}{l}\text { Öffent- } \\
\text { lichkeit }\end{array}$ & $\begin{array}{l}\text { Wissen- } \\
\text { schaft }\end{array}$ & $\begin{array}{l}\text { Wirt- } \\
\text { schaft }\end{array}$ & $\begin{array}{c}\text { Verwal- } \\
\text { tung }\end{array}$ \\
\hline - Bedarfsanalyse & $\mathrm{x}$ & $\mathrm{x}$ & $\mathrm{x}$ & $\mathrm{x}$ \\
\hline - Marktanalysen & $\mathrm{x}$ & $\mathrm{x}$ & $\mathrm{x}$ & $\mathrm{x}$ \\
\hline - Wirtschaftlichkeitsberechnung & $(\mathrm{x})$ & $(\mathrm{x})$ & $(\mathrm{x})$ & $\mathrm{x}$ \\
\hline \multicolumn{5}{|l|}{ Ausschreibung } \\
\hline \multirow{14}{*}{$\begin{array}{l}\text { - Beschreibung der Ausschreibung } \\
\text { - Vergabestelle/Auftraggeber } \\
\text { - Ort der Leistungserbringung } \\
\text { - Verfahrensart } \\
\text { - Vergabeverfahren/Vertragsart } \\
\text { - Eignungskriterien } \\
\text { - Zuschlagskriterien } \\
\text { - Teilnahmefrist } \\
\text { - Angebotsfrist } \\
\text { - Ausschreibungsunterlagen } \\
\text { - Einkaufsrichtlinien und Gesetze } \\
\text { - Einkaufsmethode } \\
\text { - Vertragslaufzeit } \\
\text { - Basisinformationen Mitbietende }\end{array}$} & $\mathrm{x}$ & $\mathrm{x}$ & $\mathrm{x}$ & $\mathrm{x}$ \\
\hline & $\mathrm{x}$ & $\mathrm{x}$ & $\mathrm{x}$ & $\mathrm{x}$ \\
\hline & $\mathrm{x}$ & $\mathrm{x}$ & $\mathrm{x}$ & $\mathrm{x}$ \\
\hline & $\mathrm{x}$ & $\mathrm{x}$ & $\mathrm{x}$ & $\mathrm{x}$ \\
\hline & $\mathrm{x}$ & $\mathrm{x}$ & $\mathrm{x}$ & $\mathrm{x}$ \\
\hline & $\mathrm{x}$ & $\mathrm{x}$ & $\mathrm{x}$ & $\mathrm{x}$ \\
\hline & $\mathrm{x}$ & $\mathrm{x}$ & $\mathrm{x}$ & $\mathrm{x}$ \\
\hline & $\mathrm{x}$ & $\mathrm{x}$ & $\mathrm{x}$ & $\mathrm{x}$ \\
\hline & $\mathrm{x}$ & $\mathrm{x}$ & $\mathrm{x}$ & $\mathrm{x}$ \\
\hline & $\mathrm{x}$ & $\mathrm{x}$ & $\mathrm{x}$ & $\mathrm{x}$ \\
\hline & $\mathrm{x}$ & $\mathrm{x}$ & $\mathrm{x}$ & $\mathrm{x}$ \\
\hline & $\mathrm{x}$ & $\mathrm{x}$ & $\mathrm{x}$ & $\mathrm{x}$ \\
\hline & $\mathrm{x}$ & $\mathrm{x}$ & $\mathrm{x}$ & $\mathrm{x}$ \\
\hline & Nein & $(\mathrm{x})^{*}$ & $(\mathrm{x})^{*}$ & $\mathrm{x}$ \\
\hline \multicolumn{5}{|l|}{ Bewertung } \\
\hline \multirow{3}{*}{$\begin{array}{l}\text { - Eignung der Mietbietenden } \\
\text { - Anzahl/Höhe eingeg. Angebote } \\
\text { - Gewichtung der Zuschlagskriterien }\end{array}$} & Nein & $(\mathrm{x})^{*}$ & Nein & $\mathrm{x}$ \\
\hline & $(\mathrm{x})^{*}$ & $(\mathrm{x})^{*}$ & $(\mathrm{x})^{*}$ & $\mathrm{x}$ \\
\hline & $\mathrm{x}$ & $\mathrm{x}$ & $\mathrm{x}$ & $\mathrm{x}$ \\
\hline \multicolumn{5}{|l|}{ Vergabe/Vertrag } \\
\hline \multirow{4}{*}{$\begin{array}{l}\text { - Finaler Vertrag } \\
\text { - Finaler Lieferant } \\
\text { - Zuschlagshöhe/Vertragsvolumen } \\
\text { - Finale Konditionen/Zahlungsbed. }\end{array}$} & $(\mathrm{x})^{*}$ & $(\mathrm{x})^{*}$ & $(\mathrm{x})^{*}$ & $\mathrm{x}$ \\
\hline & $\mathrm{x}$ & $\mathrm{x}$ & $\mathrm{x}$ & $\mathrm{x}$ \\
\hline & $\mathrm{x}^{*}$ & $(\mathrm{x})^{*}$ & $(\mathrm{x})^{*}$ & $\mathrm{x}$ \\
\hline & Nein & $(\mathrm{x})^{*}$ & $(\mathrm{x})^{*}$ & $\mathrm{x}$ \\
\hline \multicolumn{5}{|l|}{ Ausführung } \\
\hline \multirow{7}{*}{$\begin{array}{l}\text { - Vertragsänderungen/Ergebnisse } \\
\text { - Bewertung der Leistungserbringung } \\
\text { - Gesamtdokumentation } \\
\text { - Überprüfung Business Case } \\
\text { - Mängel-/Gewährleistungsverfahren } \\
\text { - Zahlungsverkehr/Abrechnung } \\
\text { - Ergebnisse von Audits, Stichproben }\end{array}$} & $(\mathrm{x})^{*}$ & $(\mathrm{x})^{*}$ & $(\mathrm{x})^{*}$ & $\mathrm{x}$ \\
\hline & $(\mathrm{x})$ & $(\mathrm{x})$ & $(\mathrm{x})$ & $\mathrm{x}$ \\
\hline & Nein & Nein & Nein & $\mathrm{x}$ \\
\hline & Nein & $(\mathrm{x})$ & Nein & $\mathrm{x}$ \\
\hline & Nein & Nein & Nein & $\mathrm{x}$ \\
\hline & Nein & Nein & Nein & $\mathrm{x}$ \\
\hline & Nein & $(\mathrm{x})$ & Nein & $\mathrm{x}$ \\
\hline $\mathrm{x}$ & \multirow{4}{*}{\multicolumn{4}{|c|}{$\begin{array}{lc}\text { Zugang } & >49 \% \\
\text { Teils-teils } & 35-49 \% \\
\text { Kein Zugang } & <35 \% \\
\text { Ggf. Schutz personenbezogener Daten/ } \\
\text { Geschäftsgeheimnisse }\end{array}$}} \\
\hline$(\mathrm{x})$ & & & & \\
\hline Nein & & & & \\
\hline & & & & \\
\hline
\end{tabular}




\subsubsection{Partizipation und Kollaboration}

\section{Nutzung eines erweiterten Projektteams sowie Erarbeitung einer Partizipations- und Kollaborationsstrategie}

Bezüglich Partizipation und Kollaboration empfiehlt es sich, neben einem Projekt-Kernteam einen erweiterten Kreis an Anwendern der verschiedenen Zielgruppen einzuladen. Gemeinsam sollte eine Partizipations- und Kollaborationsstrategie erarbeitet werden, die die verschiedenen Werkzeuge/ Mechanismen und ihre Ziele darlegt. Wichtig ist es, über den Gesamtverlauf des Projektes konkrete Mitarbeit und Feedback zu ermöglichen, aber auch die Weiterverwendung der öffentlichen Einkaufsdaten innerhalb und außerhalb der eigenen Organisationen zu erhöhen (Mendes \& Fazekas, 2018). Dies fußt auf der Erkenntnis, dass die transparente Bereitstellung von Daten nicht zwangsläufig zu einer Nutzung führt (Granickas, 2016, S. 10). So kann unter anderem Feedback zu den gewünschten und benötigten Daten (Nachfrage- und Anbietersicht), zu ihrer Priorisierung, zur Einfachheit der Analysemöglichkeiten, zur Vollständigkeit, zu Qualität und Weiterverwendungsmöglichkeiten der Daten (Arbeitskreis Open Government Partnership Deutschland, 2017, S. 42; Mendes \& Fazekas, 2018, S. 21) oder der Bedienerfreundlichkeit des Portals erhoben werden.

Gleichzeitig sollten diese Mechanismen dazu genutzt werden, dass Dritte konkrete öffentliche Einkaufsentscheidungen begleiten. In der Studie sprechen sich $53 \%$ der Befragten für die Einbeziehung ,,neutraler Dritter“ in den Auswahlprozess und $47 \%$ für ein frühes Engagement in der Nutzenanalyse/Machbarkeitsstudie aus (siehe Abschnitt 8.2.3, Frage 28). Die Formen können dabei ebenfalls unterschiedlich sein. Sie können von persönlichen über elektronische bis hin zu offenen Partizipations- und Kollaborationsformen oder einer Kombination dieser reichen. Von Lucke schlägt zum Beispiel die Einrichtung von Fachgruppen zum offenen Vergabewesen möglichst aller föderalen Ebenen oder einen Arbeitskreis zwischen Verwaltung und NGOs vor (Arbeitskreis Open Government Partnership Deutschland, 2017, S. 42).

15. Identifikation von Informations- und Schulungsbedarfen für die Öffentlichkeit und Entwicklung entsprechender Angebote

Im Handlungsfeld 15 sollte man die Anforderungen an Informationen und Schulungen aufnehmen, um so die öffentlichen Einkaufsdaten und ihre Verwendung im Prozess zu erklären, die Datenstruktur und -formate zu erörtern, Auswertungs- und Visualisierungswerkzeuge sowie Feedbackmöglichkeiten vorzustellen. Diese Schulungen können ebenfalls über verschiedene Formate bereit- 
gestellt werden (zum Beispiel Online-Schulungen, Recordings). Sie sollen einer mangelnden Nutzung und falschen Interpretation der Daten entgegenwirken, und zwar in Bezug auf alle Akteure. Dies gewinnt umso mehr an Bedeutung, da immer mehr Daten bereitstehen. Auch hier gilt es, klar abzugrenzen, welche Daten mit welchen Methoden (zum Beispiel technisch-statistisch, kulturell, strategisch, intuitiv, narrativ) ausgewertet werden. Eine rein technisch-statistische Auswertung mag für einige Daten sinnvoll sein, in bestimmten Fällen aber durch andere Verfahren ergänzt werden (Hill, 2014, S. 215 ff.). Die Studie unterstreicht ebenfalls den Qualifizierungsbedarf (siehe Abschnitt 8.2.2, Frage 24).

\subsection{Zeitliche Umsetzung}

Um eine Einschätzung zur zeitlichen Umsetzbarkeit zu erhalten, wurden die Handlungsfelder in ein Raster aus „Komplexität der Umsetzung“ und „Nutzen“ mit Blick auf die Öffnung der öffentlichen Einkaufsdaten eingeordnet (siehe Abbildung 10.5). Mit einem mittleren bis hohen Nutzen, aber begleitet von einer hohen Umsetzungskomplexität, erscheinen die Handlungsfelder, die rechtliche und organisatorische Veränderungen zum Ziel haben. Hier bedarf es aufgrund der föderalen Strukturen und verschiedenen Akteure umfänglicher Abstimmungsrunden. Dies wiederum setzt neben Fachwissen eine hohe Führungskompetenz voraus. Hingegen wird eine niedrigere bis mittlere Umsetzungskomplexität bei Themen angenommen, die klarere Arbeitsergebnisse besitzen und insbesondere eine hohe Fachexpertise erfordern.

Hieran zeigt sich, dass es zwar einzelne Handlungsfelder gibt, die bei geringer Komplexität mindestens einen mittleren Nutzen aufzeigen, diese aber dennoch nicht losgelöst von den anderen Handlungsfeldern vorgezogen und umgesetzt werden könnten. So ist die Schaffung von Partizipations- und Kollaborationsmöglichkeiten (15) sicherlich leichter umsetzbar, würde aber ohne die Vorarbeiten in anderen Handlungsfeldern keinen Nutzen stiften. Das bedeutet, dass Abhängigkeiten zwischen den einzelnen Handlungsfeldern bestehen, die erarbeitet und berücksichtigt werden müssen.

Zeitlich ergibt sich für die Aufteilung von Zielen und Aktivitäten ein sinnvoller Zeithorizont von kurzfristig (1-3 Jahre), mittelfristig (3-5 Jahre) und langfristig ( $>5$ Jahre). Dies erfolgt in Anlehnung an die Einschätzung der Teilnehmer der Studie, die mehrheitlich davon ausgehen, dass die Öffnung des öffentlichen Einkaufs nicht mehr als fünf Jahre benötigen wird (siehe Abschnitt 8.2.3).

Die Empfehlung lautet, wie in Abbildung 10.6 dargestellt, kurzfristig einerseits die Basis zu schaffen und andererseits die Öffnung öffentlicher Einkaufs- 

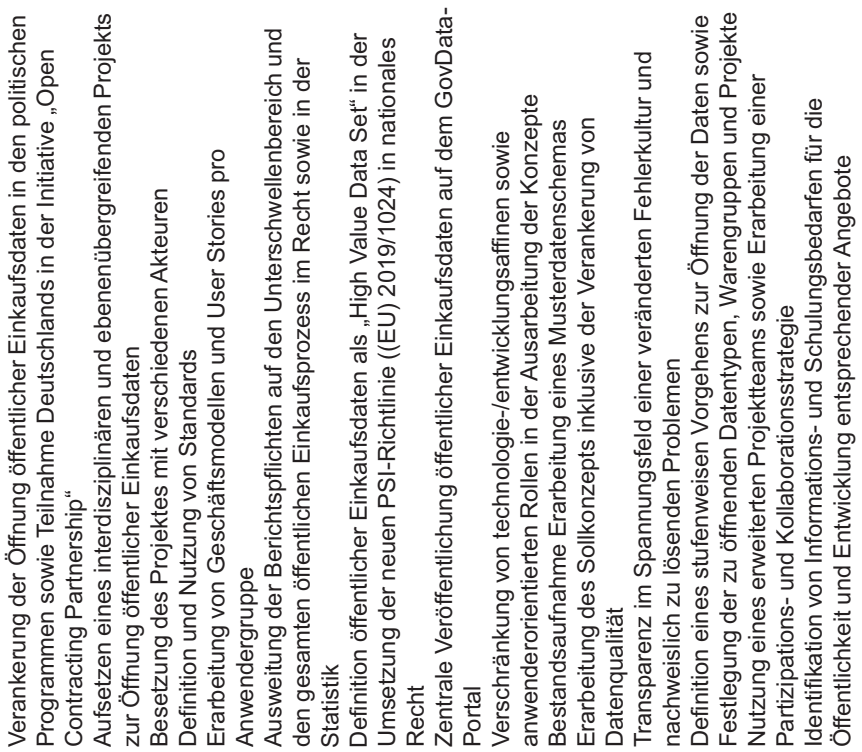

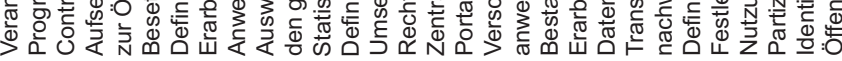

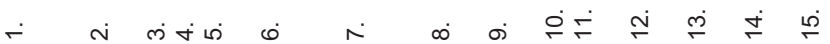

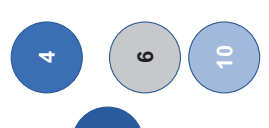

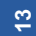



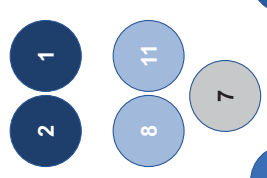

(n)

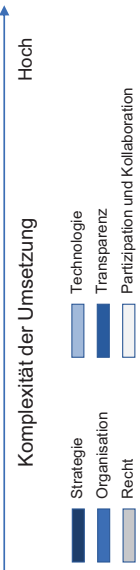

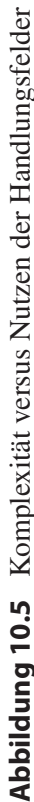

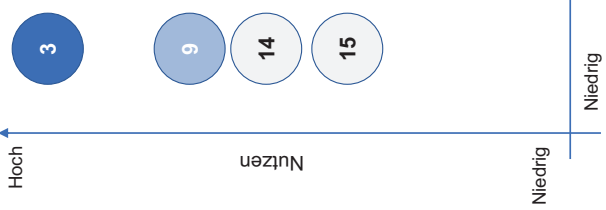




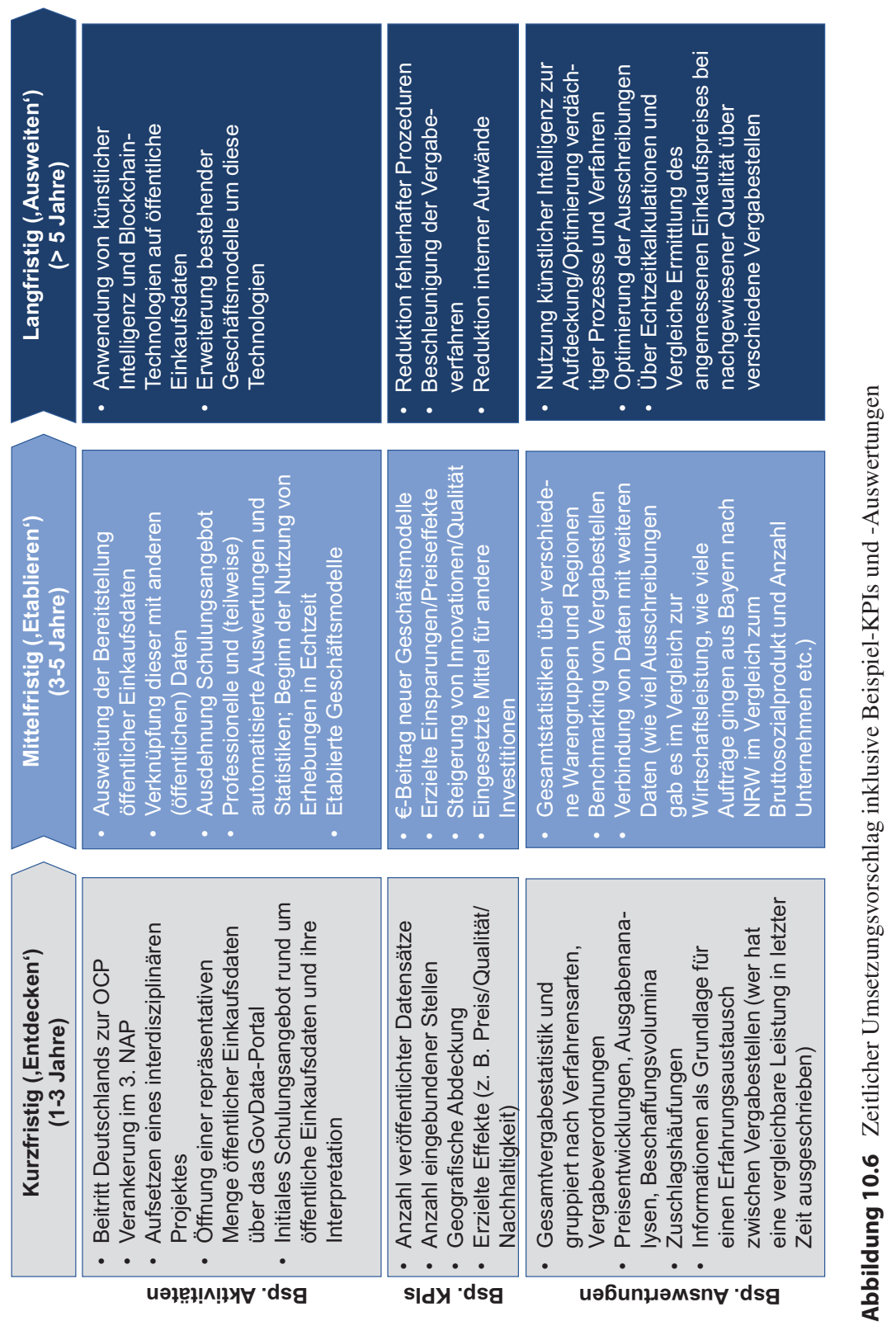


daten zu „entdecken“, sich dieses komplexe Thema also nach und nach zu erarbeiten. Hierzu gehört als Ergebnis allerdings auch die Bereitstellung einer repräsentativen Menge öffentlicher Einkaufsdaten über das GovData-Portal. Wie dies geschieht, muss dann festgelegt werden (zum Beispiel über Steuerung des Datenumfangs oder des Vorgehens; gegebenenfalls Beginn mit einzelnen Modellkommunen/Piloten und später flächige Einbindung weiterer Organisationseinheiten und Teilnehmer). Mittelfristig sollte sich die Bereitstellung öffentlicher Einkaufsdaten etablieren. Linked Open Public Procurement Data sollten verfügbar sein (siehe Abschnitt 7.3.2.2). Neue Geschäftsmodelle sind bereits am Markt vorhanden und bekannt. Langfristig, hier jenseits des 5-Jahreszeitraums, sollten Technologien wie KI und Blockchain Eingang in den Umgang mit öffentlichen Einkaufsdaten gefunden haben.

\subsection{Zwischenfazit: Potentiale eines offenen öffentlichen Einkaufs über Handlungsfelder aktiv erschließbar}

Die Definition einer Vision sowie die Erarbeitung konkreter Handlungsfelder sind hilfreich, um die Öffnung öffentlicher Einkaufsdaten genauer zu bewerten und die Umsetzung zeitlich einzuordnen. Eine pauschale Befürwortung der Öffnung ist genauso fragwürdig wie pauschale Ablehnung: Die Komplexität des Themas erfordert eine mehrschichtige Betrachtung aus verschiedenen Blickwinkeln; allerdings darf dies nicht zulasten konkreter Ergebnisse gehen. Neue Ansätze wie agile Arbeitsweisen bieten interessante Lösungsansätze, um diesen und anderen Herausforderungen zu begegnen und die mit der Öffnung der öffentlichen Einkaufsdaten verbundenen Chancen zu nutzen. Ein abschließender Blick auf die ganze vorliegende Untersuchung mit ihren Teilergebnissen wird hierzu eine Gesamteinschätzung vornehmen. 
Open Access Dieses Kapitel wird unter der Creative Commons Namensnennung 4.0 International Lizenz (http://creativecommons.org/licenses/by/4.0/deed.de) veröffentlicht, welche die Nutzung, Vervielfältigung, Bearbeitung, Verbreitung und Wiedergabe in jeglichem Medium und Format erlaubt, sofern Sie den/die ursprünglichen Autor(en) und die Quelle ordnungsgemäß nennen, einen Link zur Creative Commons Lizenz beifügen und angeben, ob Änderungen vorgenommen wurden.

Die in diesem Kapitel enthaltenen Bilder und sonstiges Drittmaterial unterliegen ebenfalls der genannten Creative Commons Lizenz, sofern sich aus der Abbildungslegende nichts anderes ergibt. Sofern das betreffende Material nicht unter der genannten Creative Commons Lizenz steht und die betreffende Handlung nicht nach gesetzlichen Vorschriften erlaubt ist, ist für die oben aufgeführten Weiterverwendungen des Materials die Einwilligung des jeweiligen Rechteinhabers einzuholen.

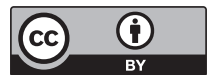

